\title{
Calculation of Elliptic Integrals of the Third Kind by Means of Gauss' Transformation
}

\author{
By Henry E. Fettis
}

1. Introduction. The elliptic integral of the third kind may be defined as

$$
\Pi\left(\theta, \alpha^{2}, k\right)=\int_{0}^{\theta} \frac{d \phi}{\left(1-\alpha^{2} \sin ^{2} \phi\right) \sqrt{ }\left(1-k^{2} \sin ^{2} \phi\right)}
$$

$(0 \leqq \theta \leqq \pi / 2) ;-\infty<\alpha^{2}<\infty ; 0 \leqq k^{2}<1$. When $\theta=\pi / 2$, it is known that the above integral can be expressed in terms of elliptic integrals of the first and second kind, respectively [1]. For other values of $\theta$, the integral can be expanded in series involving theta functions. Because of their high complexity, these are seldom used as a means of computation. Recourse is usually made to evaluation of the integral by quadrature, or sometimes by numerically integrating the differential equation which is satisfied by $\Pi\left(\theta, \alpha^{2}, k\right)$ when the latter is regarded as a function of $k$. A combination of these last mentioned methods was employed by Selfridge and Maxfield [2] in compiling their extensive tables. Clearly either one is wholly unsuitable if (for example) a computer subroutine for obtaining $\Pi\left(\theta, \alpha^{2}, k\right)$ were required, and even the representation by means of theta functions would probably be much too clumsy to use as a means of generating the function directly.

The method presented in the present paper, based on Gauss' modulus-reducing transformation, enables one to write an efficient program for direct computation of the integral for any given values of its three arguments.

Numerous methods using this and similar transformations have been proposed as a means of computing integrals of the first and second kind, [3], [4], [5], but the utility of Gauss' transformation as a practical tool for computing integrals of the third kind appears to have been overlooked.

The basic idea is to express the original integral in terms of one with a smaller value of the modulus together with perhaps elementary functions, and to apply the transformation repeatedly until the modulus is sufficiently small for the integral to be evaluated in terms of elementary functions. The value of the original integral may then be found by reversing the order of the transformations or, alternatively, by developing a recurrénce relationship which enables one to express the original integral directly in terms of elementary functions.

2. Gauss' Transformation Defined. The basic relations to be used are listed below in a form suitable for routine computation. Since all of the formulae or their equivalents can be found elsewhere (see, e.g., [1]. Art. 164.02), their derivations will not be given here.

Defining

$$
F(\theta, k)=\int_{0}^{\theta} \frac{d \phi}{\sqrt{ }\left(1-k^{2} \sin ^{2} \phi\right)}
$$

Received July 1, 1964. Revised August 10, 1964. 
and

$$
\Pi\left(\theta, \alpha^{2}, k\right)=\int_{0}^{\theta} \frac{d \phi}{\left(1-\alpha^{2} \sin ^{2} \phi\right) \sqrt{ }\left(1-k^{2} \sin ^{2} \phi\right)},
$$

it can be shown that

$$
\begin{aligned}
& F(\theta, k)=\left(1+k_{1}\right) F\left(\theta_{1}, k_{1}\right), \\
& \Pi\left(\theta, \alpha^{2}, k\right)=\frac{1}{\rho}\left\{2\left(1+k_{1}\right) \Pi\left(\theta_{1}, \alpha_{1}^{2}, k_{1}\right)+\left(1+k_{1}\right)(\rho-1) F\left(\theta_{1}, k_{1}\right)\right. \\
&\left.-\Pi\left(\theta, \alpha^{2}, 0\right)\right\},
\end{aligned}
$$

where

$$
\begin{aligned}
k^{\prime} & =\mathfrak{V}\left(1-k^{2}\right), \\
\rho & =\sqrt{ }\left(1-k^{2} / \alpha^{2}\right), \\
k_{1} & =\frac{1-k^{\prime}}{1+k^{\prime}},
\end{aligned}
$$

$$
\sin \theta_{1}=\frac{\left(1+k^{\prime}\right) \sin \theta}{1+\sqrt{ }\left(1-k^{2} \sin ^{2} \theta\right)}
$$

$$
\alpha_{1}^{2}=\alpha^{2}\left(\frac{1+\rho}{1+k^{\prime}}\right)^{2}
$$

and

$$
\Pi\left(\theta, \alpha^{2}, 0\right)= \begin{cases}\frac{1}{\sqrt{ }\left(1-\alpha^{2}\right)} \tan ^{-1}\left(\sqrt{ }\left(1-\alpha^{2}\right) \tan \theta\right), & \alpha^{2}<1, \\ \tan \theta, & \alpha^{2}=1, \\ \frac{1}{\sqrt{ }\left(\alpha^{2}-1\right)} \ln \sqrt{ }\left|\frac{1+\sqrt{ }\left(\alpha^{2}-1\right) \tan \theta}{1-\sqrt{ }\left(\alpha^{2}-1\right) \tan \theta}\right|, & \alpha^{2}>1 .\end{cases}
$$

(For $\alpha^{2}>1, \Pi\left(\theta, \alpha^{2}, k\right)$ is defined to be the Cauchy principal value of the resulting divergent integral when $\theta>\sin ^{-1}(1 / \alpha)$.)

In order that Eq. 5 define a real transformation, it is necessary that $\rho$ be real. Clearly, this can happen in three of four possible cases, namely :

Case 1. $\alpha^{2}<0$;

Case 2. $\alpha^{2} \geqq 1$;

Case 3. $k^{2}<\alpha^{2} \leqq 1$.

But, in addition, it can be shown that in each of these three cases the new values of $\rho$ and $k$ will also fall into the same range, and therefore, that the transformation may be applied successively until a modulus of sufficiently small magnitude has been reached. We consider each of these in turn, after first noting that

$$
\rho_{1}=\sqrt{ }\left(1-\frac{k_{1}^{2}}{\alpha_{1}^{2}}\right)=\sqrt{ }\left(1-k_{1}\left(\frac{1-\rho}{1+\rho}\right)\right) .
$$

Case I. $\alpha^{2}<0$. 
In this case, it is clear that $\alpha_{1}{ }^{2}<0$ and, hence, a sequence of real $\rho_{i}$ and $\alpha_{i}{ }^{2}$ will always result.

Case II. $\alpha^{2} \geqq 1$.

Here, since $\left(1-k^{2} / \alpha^{2}\right) \geqq k^{\prime 2}, \rho \geqq k^{\prime}$ and, therefore, $\alpha_{1}^{2} \geqq \alpha^{2} \geqq 1$ so the sequence will again remain real.

Case III. $0<k^{2} \leqq \alpha^{2}$.

In this case, since $k^{2} / \alpha^{2} \leqq 1$ and $\rho<1$, it follows that

$$
k_{1}{ }^{2} / \alpha_{1}{ }^{2}=k_{1}(1-\rho) /(1+\rho) \leqq 1 .
$$

Thus, all subsequent $\rho_{i}, \alpha_{i}$ will be real.

In the remaining

Case IV. $0<\alpha^{2} \leqq k^{2}$,

The transformation can not conveniently be applied, since complex values will result in the subsequent transformations. However, since $k^{2} / \alpha^{2} \geqq 1, \Pi\left(\theta, k^{2} / \alpha^{2}, k\right)$ may be found by employing the transformation applicable to Case II, and the desired result obtained by using the following known identities [1, Art. 117.02]:

$$
\begin{aligned}
\Pi\left(\theta, \alpha^{2}, k\right)+\Pi\left(\theta, k^{2} / \alpha^{2}, k\right) & =F(\theta, k)+\frac{1}{\sqrt{ }\left(\left(1-\alpha^{2}\right)\left(1-k^{2} / \alpha^{2}\right)\right)} \tan ^{-1} U, \\
U & =\sqrt{\left(\frac{\left(1-\alpha^{2}\right)\left(1-k^{2} / \alpha^{2}\right)}{1-k^{2} \sin ^{2} \theta}\right) \tan \theta ;} \\
\Pi\left(\theta, \alpha^{2}, k\right)+\Pi\left(\theta, k^{2} / \alpha^{2}, k\right) & =F(\theta, k)+\frac{\alpha^{2}<0, \text { or } \quad k^{2}<\alpha^{2}<1 ;}{2 \sqrt{ }\left(\left(1-\alpha^{2}\right)\left(1-k^{2} / \alpha^{2}\right)\right)} \\
& \cdot \ln \left|\frac{1+U}{1-U}\right|, \\
U & =\sqrt{\left(\frac{\left(1-\alpha^{2}\right)\left(k^{2} / \alpha^{2}-1\right)}{1-k^{2} \sin ^{2} \theta}\right) \tan \theta ;} \alpha^{2}>1, \text { or } 0<\alpha^{2}<k^{2} .
\end{aligned}
$$

3. The Case $\alpha^{2}>1$. When $\alpha^{2}>1$, the function $\Pi\left(\theta, \alpha^{2}, k\right)$ is singular for $\sin \theta=1 / \alpha$, and for $\theta>\sin ^{-1}(1 / \alpha)$ is defined by the Cauchy principal value of the resulting divergent integral. Since it is easier to work with quantities which remain finite, we introduce, in place of $\Pi\left(\theta, \alpha^{2}, k\right)$, the function

$$
\bar{\Pi}\left(\theta, \alpha^{2}, k\right)=\Pi\left(\theta, \alpha^{2}, k\right)-\frac{1}{\rho} \Pi\left(\theta, \alpha^{2}, 0\right),
$$

and rewrite Eq. (5) as

$$
\begin{aligned}
& \bar{\Pi}\left(\theta, \alpha^{2}, k\right) \\
& =\frac{1}{\rho}\left\{2\left(1+k_{1}\right) \bar{\Pi}\left(\theta_{1}, \alpha_{1}{ }^{2}, k_{1}\right)+(\rho-1)\left(1+k_{1}\right) F\left(\theta_{1}, k_{1}\right)-H\left(\theta, \theta_{1}, \alpha^{2}, \alpha_{1}{ }^{2}\right)\right\}
\end{aligned}
$$

where

$$
H\left(\theta, \theta_{1}, \alpha^{2}, \alpha_{1}^{2}\right)=2\left[\Pi\left(\theta, \alpha^{2}, 0\right)-\frac{1+k_{1}}{\rho_{1}} \Pi\left(\theta_{1}, \alpha_{1}^{2}, 0\right)\right]
$$


Writing $U\left(\theta, \alpha^{2}\right)=\sqrt{ }\left(\alpha^{2}-1\right) \tan \theta$, this becomes

$$
\begin{aligned}
& H\left(\theta, \theta_{1}, \alpha^{2}, \alpha_{1}^{2}\right) \\
& \quad=\frac{1}{\sqrt{ }\left(\alpha^{2}-1\right)} \ln \left|\frac{1+U\left(\theta, \alpha^{2}\right)}{1-U\left(\theta, \alpha^{2}\right)}\right|-\frac{1+k_{1}}{\rho_{1} \sqrt{ }\left(\alpha_{1}^{2}-1\right)} \ln \left|\frac{1+U\left(\theta_{1}, \alpha_{1}^{2}\right)}{1-U\left(\theta_{1}, \alpha_{1}^{2}\right)}\right|,
\end{aligned}
$$

and one can easily verify that

$$
\frac{1+k_{1}}{\rho_{1}}=\frac{\sqrt{ }\left(\alpha_{1}^{2}-1\right)}{\sqrt{ }\left(\alpha^{2}-1\right)} .
$$

Hence

$$
H\left(\theta, \theta_{1}, \alpha^{2}, \alpha_{1}^{2}\right)=\frac{1}{\sqrt{ }\left(\alpha^{2}-1\right)}\left\{\ln \left|\frac{\left[1+U\left(\theta, \alpha^{2}\right)\right]\left[1-U\left(\theta_{1}, \alpha_{1}^{2}\right)\right]}{\left[1+U\left(\theta_{1}, \alpha_{1}^{2}\right)\right]\left[1-U\left(\theta, \alpha^{2}\right)\right]}\right|\right\} .
$$

The expression (19) remains finite when $\theta \rightarrow \sin ^{-1}(1 / \alpha), \theta_{1} \rightarrow \sin ^{-1}\left(1 / \alpha_{1}\right)$, but assumes an indeterminate form. However, it is easily transformed in such a way that it becomes determinate by multiplying numerator and denominator by

$$
\left[1+U\left(\theta_{1}, \alpha_{1}^{2}\right)\right]\left[1+U\left(\theta, \alpha^{2}\right)\right]
$$

and simplifying:

$$
\begin{aligned}
& H\left(\theta, \theta_{1}, \alpha^{2}, \alpha_{1}^{2}\right) \\
& \quad=\frac{1}{\sqrt{ }\left(\alpha^{2}-1\right)}\left\{\ln \frac{\left[\cos \theta_{1} \sqrt{ }\left(1-k_{1}{ }^{2} \sin ^{2} \theta_{1}\right)+\rho_{1} \sqrt{ }\left(\alpha_{1}{ }^{2}-1\right) \sin \theta_{1}\right]^{2}}{\left[\cos \theta_{1}+\sqrt{ }\left(\alpha_{1}{ }^{2}-1\right) \sin \theta_{1}\right]^{2}\left[1-\frac{k_{1}{ }^{2}}{\alpha_{1}{ }^{2}} \sin ^{2} \theta_{1}\right]}\right\} .
\end{aligned}
$$

For $\theta_{1}=\sin ^{-1}\left(1 / \alpha_{1}\right)$ this reduces simply to

$$
H\left(\theta, \theta_{1}, \alpha^{2}, \alpha_{1}^{2}\right)=\frac{1}{\sqrt{ }\left(\alpha^{2}-1\right)} \ln \left[\frac{\rho_{1}^{2}(1+\rho)^{2}}{4 \rho}\right] .
$$

The addition formula, Eq. (13), can also be rewritten so as to avoid the singular behavior when $\sin \theta=1 / \alpha$ :

$$
\begin{aligned}
& \bar{\Pi}\left(\theta, \alpha^{2}, k\right)+I\left(\theta, k^{2} / \alpha^{2}, k\right) \\
& =F(\theta, k)+\frac{1}{\rho \sqrt{ }\left(\alpha^{2}-1\right)} \ln \sqrt{ }\left|\frac{(1-U)(1+V)}{(1+U)(1-V)}\right|,
\end{aligned}
$$

where

$$
\begin{aligned}
& V=\sqrt{ }\left(\frac{\rho^{2}\left(\alpha^{2}-1\right)}{1-k^{2} \sin ^{2} \theta}\right) \tan \theta ; \\
& U=\sqrt{ }\left(\alpha^{2}-1\right) \tan \theta .
\end{aligned}
$$

By a transformation similar to that used in obtaining (20) we can show that the last term of Eq. (21) can also be written as

$$
\frac{1}{\rho \sqrt{ }\left(\alpha^{2}-1\right)} \ln \sqrt{ }\left(\frac{\left[\cos \theta \sqrt{ }\left(1-k^{2} \sin ^{2} \theta\right)+\rho \sqrt{ }\left(\alpha^{2}-1\right) \sin \theta\right]^{2}}{\left[\cos \theta+\sqrt{ }\left(\alpha^{2}-1\right) \sin \theta\right]^{2}\left(1-k^{2} / \alpha^{2} \sin ^{2} \theta\right)}\right),
$$


which for $\sin \theta=1 / \alpha$ reduces to

$$
\frac{1}{\rho \sqrt{ }\left(\alpha^{2}-1\right)} \ln \sqrt{\left(\frac{1-k^{2} / \alpha^{2}}{1-k^{2} / \alpha^{4}}\right)} \text {. }
$$

Eqs. (20) and (21) are still undefined when $\alpha=1$, but in this case it can be shown that

$$
H\left(\theta, \theta_{1}, 1,1\right)=\frac{-2 k_{1}^{2}\left(1+k_{1}\right) \sin \theta_{1} \cos \theta_{1}}{k_{1}{ }^{\prime} \sqrt{ }\left(1-k_{1}{ }^{2} \sin ^{2} \theta_{1}\right)\left[k_{1}{ }^{\prime}+\sqrt{ }\left(1-k_{1}{ }^{2} \sin ^{2} \theta_{1}\right)\right]}
$$

and that for $\alpha \rightarrow 1$ the last term of Eq. (21) becomes

$$
\frac{-k^{2} \sin \theta \cos \theta}{k^{\prime} \sqrt{ }\left(1-k^{2} \sin ^{2} \theta\right)\left[k^{\prime}+\sqrt{ }\left(1-k^{2} \sin ^{2} \theta\right)\right]} .
$$

4. Calculation of $\Pi\left(\theta, \alpha^{2}, k\right)$ by a Recurrence Procedure. Eq. (15) expresses a linear relationship between the function $\Pi\left(\theta, \alpha^{2}, k\right)$ and the same function of the parameters $\theta_{1}, \alpha_{1}{ }^{2}, k_{1}$. A similar relation can be written between $\Pi\left(\theta_{1}, \alpha_{1}{ }^{2}, k_{1}\right)$ and $\Pi\left(\theta_{2}, \alpha_{2}{ }^{2}, k_{2}\right)$ where $\theta_{2}, \alpha_{2}{ }^{2}, k_{2}$ are found from $\theta_{1}, \alpha_{1}{ }^{2}, k_{1}$ in the same way as $\theta_{1}, \alpha_{1}{ }^{2}, k_{1}$ were found from the original parameters.

In general we can write

$$
\begin{aligned}
\Pi\left(\theta_{n}, \alpha_{n}{ }^{2}, k_{n}\right)=\frac{1}{\rho_{n}}[2(1 & \left.+k_{n+1}\right) \Pi\left(\theta_{n+1}, \alpha_{n+1}^{2}, k_{n+1}\right) \\
& \left.+\left(1+k_{n+1}\right)\left(\rho_{n}-1\right) F\left(\theta_{n+1}, k_{n+1}\right)-G\left(\theta_{n}, \alpha_{n}^{2}\right)\right],
\end{aligned}
$$

where

$$
G\left(\theta_{n},{\alpha_{n}}^{2}\right)=\Pi\left(\theta_{n},{\alpha_{n}}^{2}, 0\right),
$$

and the variables with subscript $(n+1)$ come from those with subscript $(n)$ through the relations

$$
\begin{aligned}
& k_{n+1}=\frac{1-k_{n}{ }^{\prime}}{1+k_{n}{ }^{\prime}} ; \quad \alpha_{n+1}^{2}=\left(\frac{1+\rho_{n}}{1+k_{n}{ }^{\prime}}\right)^{2} \alpha_{n}{ }^{2} \\
& \rho_{n+1}=\sqrt{ }\left(1-k_{n+1}\left(\frac{1-\rho_{n}}{1+\rho_{n}}\right)\right) ; \quad \sin \theta_{n+1}=\frac{\left(1+k_{n}{ }^{\prime}\right) \sin \theta_{n}}{1+\sqrt{ }\left(1-k_{n}{ }^{2} \sin ^{2} \theta_{n}\right)} .
\end{aligned}
$$

Eq. (25) shows that the expression $\Pi\left(\theta_{1}, \alpha_{1}{ }^{2}, k_{1}\right)$ in Eq. (5) may be replaced by a corresponding relation in terms of $\Pi\left(\theta_{2}, \alpha_{2}{ }^{2}, k_{2}\right)$ and that this process may be continued so that the result after $N$ steps will be of the form

$$
\begin{aligned}
\Pi\left(\theta, \alpha^{2}, k\right) & =Q_{N} F\left(\theta_{N}, k_{N}\right)+S_{N} \Pi\left(\theta_{N}, \alpha_{N}{ }^{2}, k_{N}\right)-T_{N} ; \\
F(\theta, k) & =R_{N} F\left(\theta_{N}, k_{N}\right) .
\end{aligned}
$$

But if $N$ is sufficiently large,

$$
\begin{aligned}
k_{N} & \cong 0, \\
F\left(\theta_{N}, k_{N}\right) & \cong \theta_{N}
\end{aligned}
$$


and

$$
\Pi\left(\theta_{N}, \alpha_{N}^{2}, k_{N}\right) \cong G\left(\theta_{N}, \alpha_{N}^{2}\right)
$$

Hence, finally,

$$
\begin{aligned}
\Pi\left(\theta, \alpha^{2}, k\right) & \cong Q_{N} \theta_{N}+S_{N} G_{N}-T_{N} ; \\
F(\theta, k) & \cong R_{N} \theta_{N} .
\end{aligned}
$$

To discover the general rule for generating $Q_{n}, R_{n}, S_{n}, T_{n}$ we write

$$
\begin{aligned}
\Pi\left(\theta, \alpha^{2}, k\right) & =Q_{n} F\left(\theta_{n}, k_{n}\right)+S_{n} \Pi\left(\theta_{n},{\alpha_{n}}^{2}, k_{n}\right)-T_{n} ; \\
F(\theta, k) & =R_{n} F\left(\theta_{n}, k_{n}\right) .
\end{aligned}
$$

Substituting from Eq. (28),

$$
\begin{aligned}
& \Pi\left(\theta, \alpha^{2}, k\right)=Q_{n}\left[\left(1+k_{n+1}\right) F\left(\theta_{n+1}, k_{n+1}\right)\right] T_{n} \\
& +\frac{S_{n}}{\rho_{n}}\left[2\left(1+k_{n+1}\right) \Pi\left(\theta_{n+1}, \alpha_{n+1}^{2}, k_{n+1}\right)\right. \\
& \left.\quad+\left(1+k_{n+1}\right)\left(\rho_{n}-1\right) F\left(\theta_{n+1}, k_{n+1}\right)-G_{n}\right],
\end{aligned}
$$

or

$$
\begin{aligned}
\Pi\left(\theta, \alpha^{2}, k\right)=(1 & \left.+k_{n+1}\right)\left[Q_{n}+\frac{\rho_{n}-1}{\rho_{n}} S_{n}\right] F\left(\theta_{n+1}, k_{n+1}\right) \\
& +\frac{2\left(1+k_{n+1}\right)}{\rho_{n}} S_{n}\left[\Pi\left(\theta_{n+1}, \alpha_{n+1}^{2}, k_{n+1}\right)\right]-\left[\frac{G_{n}}{\rho_{n}} S_{n}+T_{n}\right] .
\end{aligned}
$$

It follows that, in general,

$$
\begin{array}{ll}
Q_{n+1}=\left(1+k_{n+1}\right)\left[Q_{n}+\frac{\rho_{n}-1}{\rho_{n}} S_{n}\right] ; & R_{n+1}=\left(1+k_{n+1}\right) R_{n} ; \\
S_{n+1}=\frac{2\left(1+k_{n+1}\right)}{\rho_{n}} S_{n} ; & T_{n+1}=T_{n}+\frac{G_{n}}{\rho_{n}} S_{n},
\end{array}
$$

with starting values

$$
\begin{aligned}
& Q_{0}=0, \\
& R_{0}=1, \\
& S_{0}=1, \\
& T_{0}=0 .
\end{aligned}
$$

When $\alpha^{2}>1$, the same formulae may be applied to find $\bar{\Pi}\left(\theta, \alpha^{2}, k\right)$ by replacing $G\left(\theta_{n}, \alpha_{n}\right)$ everywhere with $H\left(\theta_{n}, \theta_{n+1}, \alpha_{n}{ }^{2}, \alpha_{n+1}^{2}\right)$. In this case, since

$$
H\left(\theta_{N}, \theta_{N+1}, \alpha_{N}^{2}, \alpha_{N}^{2}+1\right) \cong 0,
$$

Eq. (29) becomes simply

$$
\bar{\Pi}\left(\theta, \alpha^{2}, k\right)=Q_{N} \theta_{N}-T_{N}
$$


Numerical Examples (Values of the parameters were chosen so as to agree with those of [2].)

$\theta=1.2, \quad k^{2}=.708073407$.

Data applicable to all cases:

\begin{tabular}{|c|c|c|c|c|c|c|}
\hline & $n$ & $k_{n}$ & $\sin \theta_{n}$ & \multicolumn{2}{|c|}{$\tan \theta_{n}$} & $R_{n}$ \\
\hline & 0 & $.70807: 3407$ & .932039086 & \multicolumn{2}{|c|}{2.572151622} & 1.000000000 \\
\hline & 1 & .089070251 & .885966451 & \multicolumn{2}{|c|}{1.910442521} & 1.298446402 \\
\hline & 2 & .000543736 & .881464024 & \multicolumn{2}{|c|}{1.866515246} & 1.328723767 \\
\hline & 3 & .000000018 & .881437292 & \multicolumn{2}{|c|}{1.866261475} & 1.328904435 \\
\hline & 4 & .000000000 & .881437901 & \multicolumn{2}{|c|}{1.866261466} & 1.328904441 \\
\hline & \multicolumn{6}{|c|}{$\theta_{N}=1.078896779, \quad F(\theta, k)=1.433750721$} \\
\hline \multirow[b]{2}{*}{$n$} & \multicolumn{6}{|c|}{$\begin{array}{l}\text { Example } 1 \\
\alpha^{2}=-1.0\end{array}$} \\
\hline & $G_{n}$ & $\rho_{n}$ & $\alpha_{n}{ }^{2}$ & $Q_{n}$ & $S_{n}$ & $T_{n}$ \\
\hline $\begin{array}{l}0 \\
1 \\
2 \\
3 \\
4\end{array}$ & $\begin{array}{l}.921017208 \\
.715170325 \\
.698905952 \\
.698810935 \\
.698810932\end{array}$ & $\begin{array}{l}1.306932824 \\
1.019660581 \\
1.000113490 \\
1.000000038 \\
1.000000000\end{array}$ & $\begin{array}{l}-1.000000000 \\
-2.243148161 \\
-2.395389266 \\
I_{2}^{2} .396312652 \\
2.396312683\end{array}$ & $\begin{array}{l}.000000000 \\
.304939790 \\
.351256386 \\
.351756787 \\
.351756819\end{array}$ & $\begin{array}{r}1.000000000 \\
1.987013223 \\
3.988281453 \\
7.976742207 \\
15.953484426\end{array}$ & $\begin{array}{r}.000000000 \\
.704716563 \\
2.098369430 \\
4.885486765 \\
10.459721422\end{array}$ \\
\hline & $\Pi\left(\alpha^{2}, \theta, k\right)=$ & $51756819)$ & $8896779)+$ & 953484426 & $8810932)-1$ & 10.459721422 \\
\hline
\end{tabular}

\begin{tabular}{cccccrrr}
\multicolumn{7}{c}{ Example 2} \\
\multicolumn{7}{c}{$\alpha^{2}=0.8$} \\
$n$ & $G_{n}$ & $\rho_{n}$ & $\alpha_{n}{ }^{2}$ & $Q_{n}$ & $S_{n}$ & \multicolumn{1}{c}{$T_{n}$} \\
0 & 1.912245968 & .338981181 & .800000000 & .000000000 & 1.000000000 & .000000000 \\
1 & 1.394007405 & .923398656 & .604542721 & -2.531991609 & 7.660876021 & 5.641156719 \\
2 & 1.361803176 & .999535557 & .585500101 & -3.241366666 & 16.979694330 & 17.206386788 \\
3 & 1.361618020 & .999999984 & .585387360 & -3.249698228 & 33.979787811 & 40.340132749 \\
4 & 1.361618014 & 1.000000000 & .585387356 & -3.249698780 & 67.959577009 & 86.607624893 \\
\multicolumn{5}{c}{$\Pi\left(\alpha^{2}, \theta, k\right)$} & $=(-3.249698780)(1.078896779)+(67.959577009)(1.361618014)-86.60762489$ \\
& $=2.421269850$.
\end{tabular}

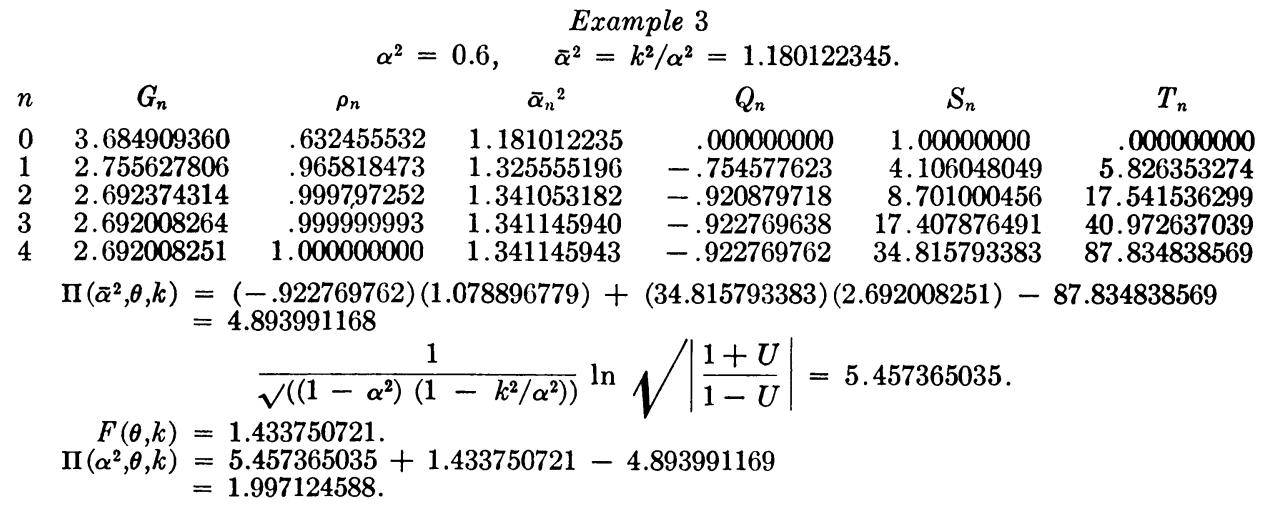

Applied Mathematics Research Laboratory

Aerospace Research Laboratories

Office of Aerospace Research

Wright-Patterson Air Force Base, Ohio 
1. Paul F. Byrd \& Morris D. Friedman, Handbook of Elliptic Integrals for Engineers and Physicists, Die Grundlehren der Mathematischen Wissenschaften in Einzeldarstellungen, Springer, Berlin, 1954.

2. R. G. SELFRIDGE \& J. E. MAXFIELD, Table of the Incomplete Elliptic Integral of the Third Kind, Dover, New York, 1958.

3. L. V. KInG, On the Direct Calculation of Elliptic Functions and Integrals, Cambridge Univ. Press, New York, 1924.

4. H. E. SALZER, "Quick calculation of the Jacobian elliptic functions," Comm. ACM, v. 5, 1962, no. 7 .

5. A. R. DiDonato \& A. V. HersheY, "New formulae for computing incomplete elliptic integrals of the first and second kind," J. Assoc. Comput. Mach., v. 6, 1959, no. 4, p. 515-526. 